OPEN ACCESS

Edited by:

Xuesong Gu,

Beth Israel Deaconess Medical Center and Harvard Medical School,

United States

Reviewed by:

Qi Pan,

Fudan University, China

Guoming Shi,

Fudan University, China

Liang Liu,

Fudan University, China

*Correspondence:

Tao $\mathrm{Li}$

litao7706@163.com

Zhiqiang Chen

chenzhq1982@hotmail.com

${ }^{t}$ These authors have contributed equally to this work and share first authorship

Specialty section:

This article was submitted to Cancer Molecular Targets and Therapeutics,

a section of the journa

Frontiers in Oncology

Received: 10 May 2021 Accepted: 08 June 2021

Published: 28 June 2021

Citation:

Xu B, Chen Z, Zhang J, Chang J,

Zhao W, Dong Z, Zhi X and Li T (2021) Prognostic Value of Peripheral

Whole Blood Cell Counts Derived Indexes in Gallbladder Carcinoma: A Systematic Review and Meta-Analysis.

Front. Oncol. 11:707742.

doi: 10.3389/fonc.2021.707742

\section{Prognostic Value of Peripheral Whole Blood Cell Counts Derived Indexes in Gallbladder Carcinoma: A Systematic Review and Meta-Analysis}

\author{
Bowen $\mathrm{Xu}^{1 \dagger}$, Zhiqiang Chen ${ }^{1 * t}$, Jing Zhang ${ }^{2}$, Jianhua Chang ${ }^{1}$, Wei Zhao ${ }^{1}$, Zhaoru Dong ${ }^{1}$, \\ Xuting $\mathrm{Zhi}^{1}$ and Tao $\mathrm{Li}^{1,3 *}$ \\ ${ }^{1}$ Department of Hepatobiliary Surgery, General Surgery, Qilu Hospital, Cheeloo College of Medicine, Shandong University, \\ Jinan, China, ${ }^{2}$ Department of Surgery, Yidu Central Hospital of Weifang City, Weifang, China, ${ }^{3}$ Department of Hepatobiliary \\ Surgery, The Second Hospital of Shandong University, Jinan, China
}

Background: Gallbladder carcinoma (GBC) is a rare gastrointestinal malignancy with poor prognosis. Adequate pre-treatment prediction of survival is essential for risk stratification and patient selection for aggressive surgery or adjuvant therapeutic strategy. Whole blood cell count (WBCC) derived indexes are broadly used as prognosticative biomarkers in various cancer types, but their utility in GBC needs to be validated.

Methods: An extensive literature review was conducted in line with PRISMA guideline until June 31 2020, to identify original studies concerning WBCC-derived indexes as prognostic indicators in GBC. All relative parameters were extracted and pooled for statistical analyses.

Results: Fourteen studies incorporating 2,324 patients were included with a high quality and low risk of biases. All 14 studies evaluated the prognostic value of NLR showing a significant correlation with $\mathrm{OS}$ in $\mathrm{GBC}$ patients $(\mathrm{HR}=1.94, P<0.001)$. Elevated NLR was revealed to correlate with TNM stage (stages III and IV, OR $=4.65, P<0.001$ ), tumor differentiation (OR $=2.37, P<0.042)$, CA 19-9 (SMD $=0.47, P=0.01)$, but no significance was found with age, sex and CEA. Positive indicative value of MLR and PLR were also confirmed with a HR of $2.06(P<0.001)$ and $1.34(P<0.001)$, respectively.

Conclusion: The WBCC-derived indexes including NLR, MLR/LMR and PLR were validated to be useful prognostic parameters for predicting survival outcomes in GBC patients. These series of indexes, especially NLR, could improve risk stratification and facilitate better patient selection for surgical resection or aggressive chemotherapy in the decision making of GBC patients.

Keywords: gallbladder carcinoma (GBC), neutrophil-to-lymphocyte ratio (NLR), lymphocyte-to-monocyte ratio (LMR), platelet-to-lymphocyte ratio (PLR), prognosis 


\section{INTRODUCTION}

Gallbladder carcinoma (GBC) is a relatively rare gastrointestinal malignancy with an estimated incidence rate about 1.0-3.0/ 100,000 in the United States and China $(1,2)$. It is unique in its characteristics that if diagnosed at an early stage (T1a and T1b, before invading beyond gallbladder mucosa), a nearly $100 \%$ 5-year survival could be achieved after surgery, but at its later stage (T2 especially $\mathrm{T} 3$ and $\mathrm{T} 4$, tumor penetrating beyond muscular layer), the long-term survival becomes dismal with only $25 \%$ of patients who could undergo potentially curative surgery and just $12-23 \%$ could survive for more than 5 years (3, 4). At present, no consensus has been achieved on the optimal treatment of GBC, and multidisciplinary therapy of surgery combined with adjuvant therapy may play a better role in prolonging the survival of patients with advanced $\operatorname{GBC}(3,5)$.

Pathologic TNM staging from the American Joint Committee on Cancer (AJCC) is now the most widely adopted accurate and effective prognosis predicting system for various cancers including GBC (6). However, it could only be assessed after surgery which may account for only a minority of patients suffering GBC. Therefore, it is necessary to pursue preoperative biological markers that could predict survival outcomes of patients, aid in risk stratification and personalized decision making on whether patient could get potential benefits from more aggressive therapies (3).

There is growing evidence that systemic inflammation response (SIR) plays an important role in cancer development and progression, thus various SIR-related biomarkers have been developed and evaluated as prognostic indicators in different cancer types $(7,8)$. One series of SIR-related biomarkers is derived from peripheral whole blood cell counts (WBCC) which include neutrophil-to-lymphocyte ratio (NLR), lymphocyte-to-monocyte ratio (LMR) or monocyte-tolymphocyte ratio (MLR), and platelet-to-lymphocyte ratio (PLR). These WBCC-derived indexes showed some great advantages over other pathology related markers, such as easy to perform, good replicability, low cost and preoperative application etc. $(7,8)$.

Despite the robust and growing data regarding the utility of these WBCC-derived indexes, findings are not uniform across all publications. Furthermore, previous studies mainly focused on colorectal, pancreatic, prostate, lung, esophageal-gastric and breast cancers (7), only few studies conducted investigations on GBC until recently (9-22). Based on the available data, we aimed to systematically review and rationalize the evidence for the prognostic value of these WBCC-derived indexes in predicting outcomes of GBC patients.

\section{MATERIALS AND METHODS}

This study was conducted in line with the PRISMA and AMSTAR guidelines that were well defined protocols for systemic reviews and meta-analysis $(23,24)$.

\section{Inclusion and Exclusion Criteria}

The inclusion criteria of studies in this meta-analysis were defined as: (i) randomized controlled trials (RCT), cohort studies or case control studies; (ii) patients diagnoses of GBC were confirmed by pathology; and (iii) any of the WBCC-derived markers (including NLR, PLR, MLR or LMR) were investigated objects of the studies, and clear cut-off values were given or could be extracted from the Kaplan-Meier curves. The exclusion criteria were: (i) basic researches or animal trials; (ii) abstracts, meta-analysis, case reports, letters, expert comments or reviews; (iii) hazard ratio (HR) investigating correlation between prognostic markers and overall survivals unavailable or cannot be extracted from the K-M curve; and (iv) patients suffered from other primary tumors or with severe infections.

\section{Search Strategy}

PubMed, Medline, Web of Science, Scopus, CNKI, and China Biology Medicine disc (CBMdisc) were searched by two independent researchers from inception to June 312020 without any other limits. The medical subject headings $(\mathrm{MeSH})$ terms and free text terms were used to locate articles, combined with the Boolean operators to make an appropriate search strategy. The MeSH terms included "Gallbladder Neoplasms", "Lymphocytes", "Neutrophils", and the free text terms included "Neutrophil-lymphocyte (Ratio)", "Neutrophil (to) Lymphocyte (Ratio)".

\section{Quality Assessment and Data Extraction}

After eliminating duplicates, two researchers read titles and abstracts, then by reading full-texts to identify eligible literatures that met the inclusion and exclusion criteria for meta-analysis. The qualitative assessment of RCTs was based on Cochrane risk of bias tool. The Newcastle-Ottawa Scale (NOS) was used to assess risk of bias in non-RCTs. Two researchers used standardized Excel sheets to extract information from the final included studies, including basic information of the study (title, first author, year of publication, study types and number of patients), demographics (patient age and gender), oncology information (tumor types, predominant treatment arms, followup time, disease-free survival (DFS), overall survival (OS), hazard ratio (HR) and 95\% confidence interval (95\% CI), NLR, PLR, LMR and other tumor markers). In the absences of vital data from a study, the corresponding author of the study was inquired by email.

\section{Statistical Analysis}

In this meta-analysis, we mainly focused on the efficacy of NLR, MLR/LMR and PLR on predicting patient survival, and HR with 95\% CI was employed to make analysis, as HR incorporates the impact of time-to-event outcomes and is more reliable to reflect survival status of patients over other statistical measures (25). Engauge Digitizer (version 10.8) and method described by Tierney et al. was used to calculate HR from available statistics and Kaplan-Meier curves if the included studies did not provide HR (26). The odds ratio (OR) was chosen to evaluate the association between NLR, MLR/LMR, PLR and clinical 
features. The numerical data were expressed as means \pm standard deviations (SD), and was calculated by using the method from Wan et al. and Luo et al., if the original studies only provided medians and interquartile ranges $(27,28)$. Heterogeneity between studies was evaluated by Chi-squared $(\chi 2)$ Q test, and the extent of heterogeneity was quantified by $\mathrm{I}^{2}$ index. The random-effects model was applied when the heterogeneity was low (I2 $<50 \%)$, otherwise, the fixed effects model was adopted (I2 >50\%). In addition, sensitivity and subgroup analyses were used to find the source of heterogeneity. The publication bias was assessed by Begg's funnel plot and Egger's test. All the statistical data were analyzed with STATA 12.0 (Version 12.0, Stata Corp LP, College Station, TX). A value of $P<0.05$ was considered statistically significant.

\section{RESULTS}

\section{Search Results and Study Characteristics}

The study selection was carried out in accordance with PRISMA flowchart (Figure 1). A total of 221 potentially relevant studies were identified from six databases by using the formulated search strategy. After removal of duplicates, we browsed the titles and abstracts of the remaining 134 studies, and 107 studies were excluded according to the inclusion and exclusion criteria. Then we assessed full-text review of the remaining 27 studies, another 13 studies were excluded and finally 14 retrospective cohort studies were included for meta-analysis (9-22). Basic characteristics of included studies were listed in Table 1. There were 2,324 patients in total from all studies with a mean NOS score of $7.6 \pm 0.69$, indicating a low risk of bias. NLR was evaluated by all the 14 studies enrolled, while MLR/LRM and PLR were investigated by only six and eight studies, respectively. Although we could calculate or directly withdraw HR data from all studies, it needs to be noted that different cut-off values were employed, as well as calculation methods for cut-off value among which ROC curve analysis was the mostly adopted by majority of authors.

\section{Prognostic Value of NLR and Its Association With Clinical Features}

NLR was evaluated in all 14 studies enrolled and HR data could be extracted directly from 14 studies and calculated from $\mathrm{K}-\mathrm{M}$ curve from one study using the method as described above (26). The heterogeneity was not significant $\left(P=0.184, \mathrm{I}^{2}=25 \%\right)$ among studies, so the fixed effect model was used for metaanalysis evaluating prognostic value between high NLR and low NLR groups. Compared with lower NRL group, higher pretreatment NLR was significantly correlated with shorter OS in GBC patients as shown in Figure 2 with a HR value of 1.94 (95\% CI=1.71-2.19, $P<0.001)$.

As most included studies investigated the association between NLR and various clinical parameters including TNM stage, tumor differentiation, CA199 and CEA etc., we then summarized the

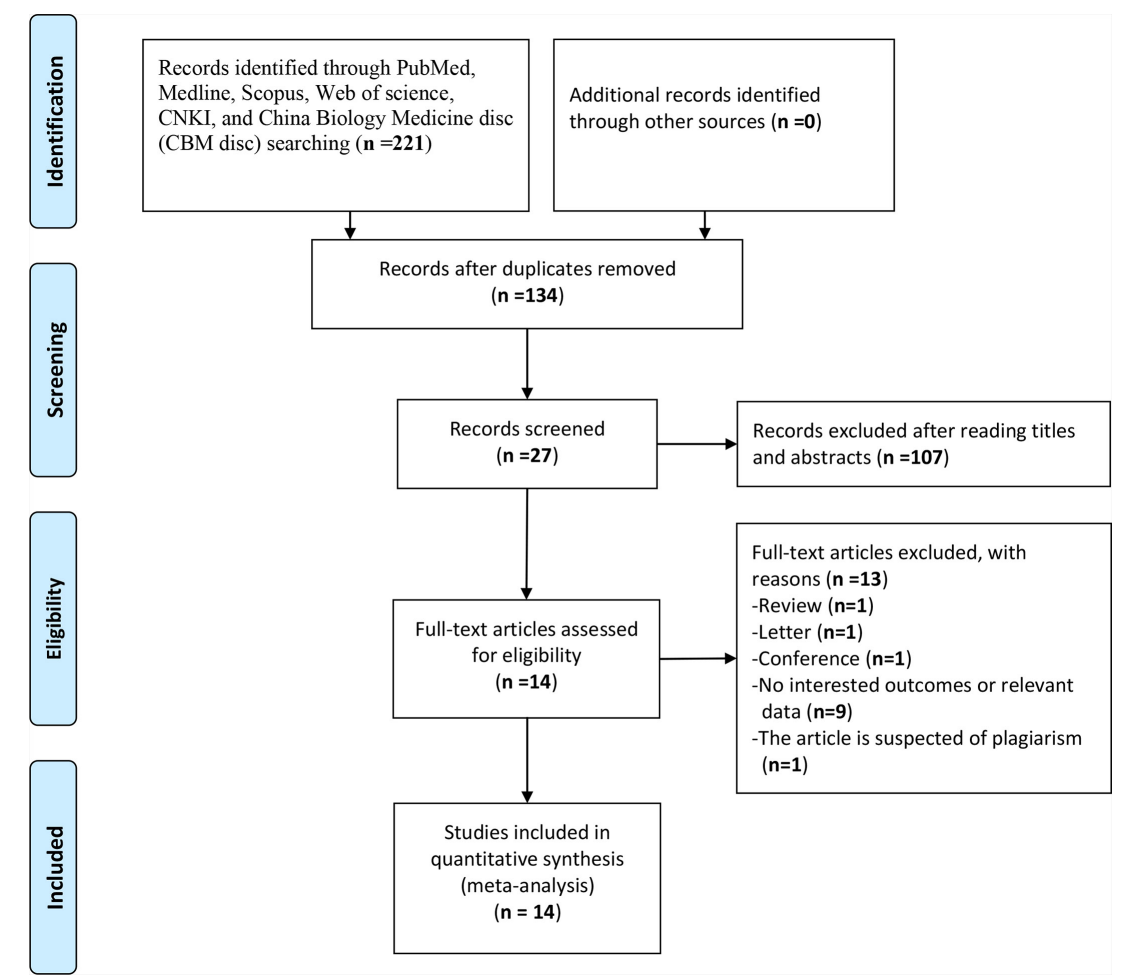

FIGURE 1 | Flow diagram following the PRISMA template of the search strategy for studies included in this meta-analysis. 
TABLE 1 | Basic characteristics of included studies.

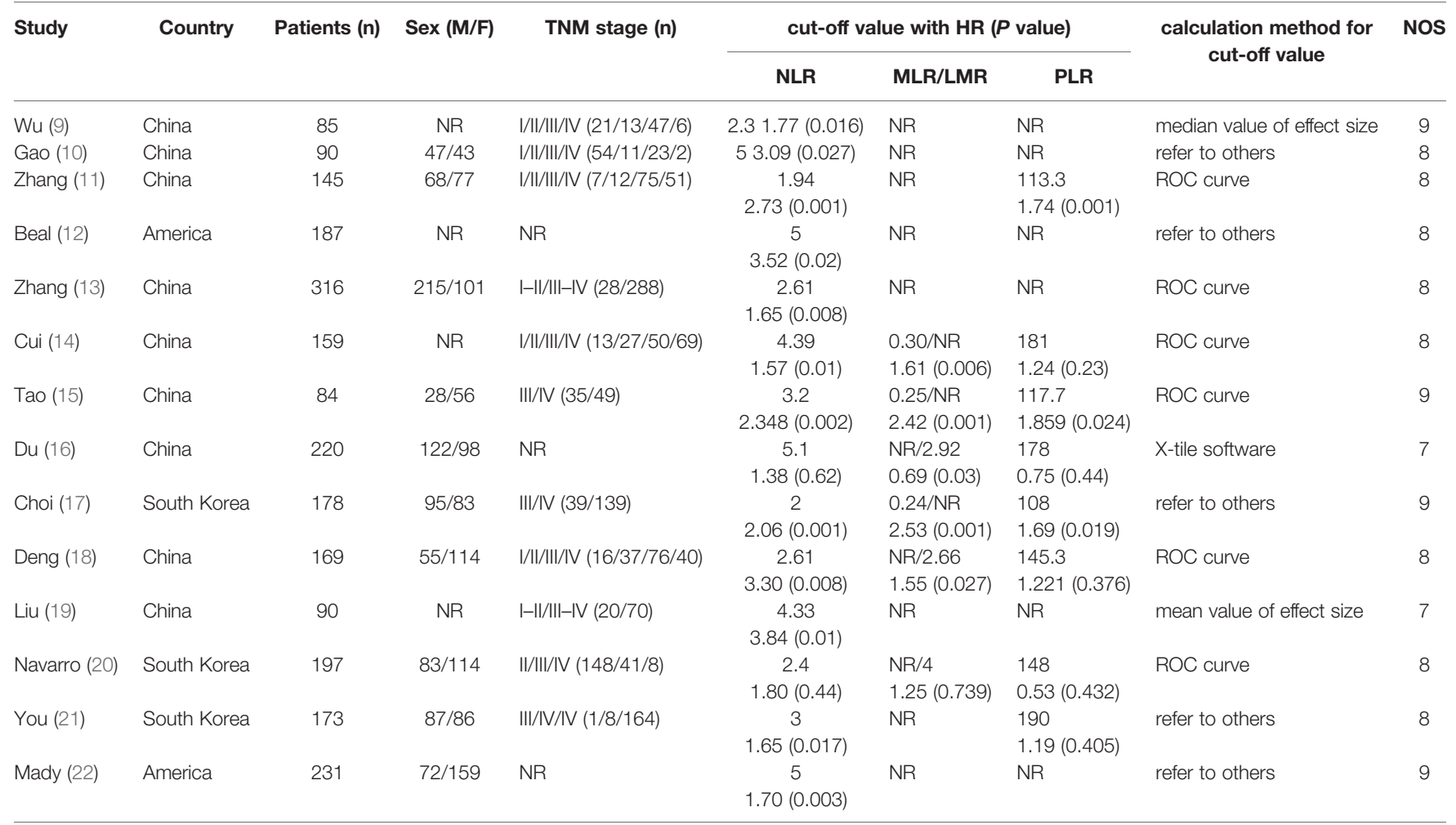

TNM, tumor/node/metastasis stages; HR, Hazard Ratio; NLR, neutrophil-to-lymphocyte ratio; MLR, monocyte-to-lymphocyte ratio; LMR, Iymphocyte-to-monocyte ratio; PLR, platelet-tolymphocyte ratio; NR, not reported; ROC, receiver operating characteristic; NOS, Newcastle-Ottawa Scale.

pooled OR of these parameters. As shown in Table 2, elevated NLR had significant correlation with TNM stage (stages III and IV, OR = $4.65,95 \% \mathrm{CI}=1.96-11.03, P<0.001)$, tumor differentiation $(\mathrm{OR}=$ 2.73, 95\% CI $=1.04-7.18, P<0.042)$, CA 19-9 $(\mathrm{SMD}=0.47,95 \%$ $\mathrm{CI}=0.11-0.82, P=0.01)$, but no significance was found with age, sex and CEA. Due to the significant heterogeneity between studies, random-effects models were used for analysis.

\section{The Prognostic Value of MLR/LMR}

Three studies incorporating 421patients reported the prognostic value of MLR in GBC patients (14-16). As shown in Figure 3, high MLR was significantly correlated with shorter OS in GBC patients compared to low MLR group, with a pooled HR of 2.06 (95\% CI $=1.51-2.82, P<0.001)$. The heterogeneity was not significant $\left(P=0.193, \mathrm{I}^{2}=39.2 \%\right)$ among studies, and the fixed effect model was used for meta-analysis. Another three studies enrolling 586 patients reported the relationship between LMR and OS in GBC patients $(17,18,21)$. The primary results showed that there was no significant correlation between LMR and prognosis of GBC patients with a HR of 1.08 (95\% CI $=0.58-2.07, P=0.814$, $\left.\mathrm{I}^{2}=70 \%\right)$. Due to the high heterogeneity, we performed sensitivity analysis and revealed that the heterogeneity decreased significantly after excluding the study by Deng et al. (17), but the final result did not change either $(\mathrm{HR}=0.73,95 \% \mathrm{CI}=0.46-$ $\left.1.15, \mathrm{I}^{2}=0 \%, P=0.176\right)$. By re-reading the study by Deng et al., we found that they set the lower LMR group as experimental group instead of control group as the other two enrolled studies
$(18,21)$. Therefore, we re-calculated the reciprocal of $\mathrm{HR}$ and $95 \%$ CI with a correction of pooled HR of 0.68 (95\% CI $=0.51-0.91$, $\left.\mathrm{I}^{2}=0 \%, P=0.011\right)$, showing significant correlation between low LMR and poor OS in GBC patients.

\section{The Prognostic Value of PLR}

Eight studies incorporating 1,325 patients investigated prognostic value of PLR in GBC patients $(11,14-18,20,21)$. No significant heterogeneity between groups was observed $\left(\mathrm{I}^{2}=\right.$ $33.4 \%, P=0.162$ ), and the pooled HR showed that low PLR group had significant better OS than high PLR group (Figure 4, $\mathrm{HR}=1.34,95 \% \mathrm{CI}=1.14-1.57, P<0.001)$.

\section{Subgroup Analyses and Publication Bias}

Our meta-analyses result above confirmed that NLR, LMR/MLR and PLR could be used as prognostic predictor of OS in GBC patients. Although there was no significant heterogeneity between groups, we still performed subgroup analysis in case of patient selection bias. Three stratification parameters were selected for subgroup analysis, that include cut-off value $(\leq 3,3-5$ and $\geq 5)$, sample size $(>100$ and $\leq 100)$ and geographic area (Asian and America). Due to sample size and data availability, the subgroup analysis was only performed in NLR group, which confirmed a positive result as ungrouped analysis (Figure 5).

In the end, we run Begg's and Egger's test to examine the main effect indicators of this study. The results showed that there was no significant publication bias among included studies, 


Study
Wu 2014
Gao 2015
Zhang 2015
Beal 2016
Zhang 2016
Cui 2018
Tao 2018
Du 2018
Choi 2019
Deng 2019
Liu 2019
Navarro 2019
You 2019
Mady 2020
Overall $(\mathrm{I}-$ squared $=25.0 \%, \mathrm{p}=0.184)$
Test of ES=1: $\mathrm{z}=10.54 \mathrm{p}=0.000$

FIGURE 2 | Forest plot for the association between neutrophil-lymphocyte ratio (NLR) and overall survival of patients with Gallbladder carcinoma (GBC).

TABLE 2 | The association between elevated neutrophil-to-lymphocyte ratio (NLR) and clinical features.

\begin{tabular}{|c|c|c|c|c|c|}
\hline Clinical parameter & $\begin{array}{l}\text { Number of } \\
\text { studies }\end{array}$ & $\begin{array}{l}\text { Number of } \\
\text { participants }\end{array}$ & \multicolumn{2}{|c|}{ Pooled results } & $P$ value \\
\hline Age (>60 years) & 2 & 180 & OR: 1.17 & $0.45-3.03$ & 0.29 \\
\hline Gender (Male) & 9 & 1,378 & OR: 1.33 & $0.95-1.87$ & 0.099 \\
\hline CEA (High) & 3 & 320 & SMD: 0.025 & $-0.198-0.249$ & 0.826 \\
\hline CA-199 (High) & 4 & 498 & SMD: 0.47 & $0.11-0.82$ & 0.01 \\
\hline
\end{tabular}

OR, odds ratio; Cl, confidence interval; CEA, carcinoembryonic antigen; CA-199, carbohydrate antigen 199; TNM, tumor/node/metastasis stage; SMD, Standard mean difference.

and the funnel plot was symmetrical (Figure 6, Begg's test $P=$ 0.373 , Egger's test $P=0.225$ ).

\section{DISCUSSION}

GBC is rare and one of the most aggressive cancers with poor prognosis worldwide (29). Up to date, pathological TNM (pTNM) staging is still the gold standard risk stratification system and reversely correlated with survival of GBC patients, but with a limitation of being only assessable after surgery (6). Surgery still remains the only potentially curative therapy, but only a minority of patients has the chance of getting radical operation and adjuvant therapy still has its position in GBC treatment $(3,30)$. Thus, efficient pre-operative or pre-treatment parameters/indexes for prediction of prognosis should be pursued, as they may help identifying patients who might benefit from more aggressive adjuvant therapies $(31,32)$.
There are some kinds of predictive parameters/indexes advocated by different authors to be used for pre-treatment evaluation of survival in various cancer types (21, 33-39). These markers were derived mainly on the basis of three major groups of clinical parameters, specifically reflecting nutrition status (glucose, albumin and cholesterol), immune status (lymphocyte, monocyte), inflammation status (neutrophil, platelet and C-reactive protein) and their cross combinations. Examples may include GLR (glucose to lymphocyte ratio) (21), CONUT (controlling nutritional status score, calculated from albumin, lymphocyte and cholesterol) (34), PNI (prognostic nutritional index, calculated from albumin and lymphocyte) (35), GPS (Glasgow prognostic score, calculated from Creactive protein and albumin) (38), SII (systemic immuneinflammation index, derived from platelet, neutrophil and lymphocyte) (39) and so on. Compared to pTNM or other pathology-based evaluators, these markers share similar advantages, such as easy to assess and replicable, low cost and 


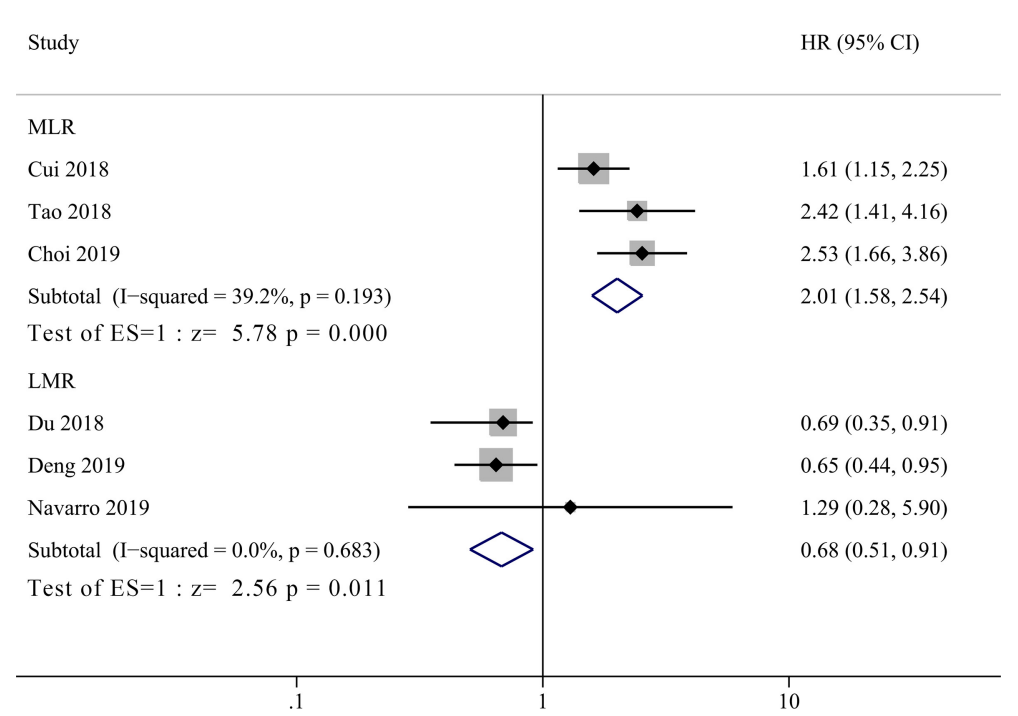

FIGURE 3 | Forest plot for the association between lymphocyte-to-monocyte ratio (LMR) or monocyte-to-lymphocyte ratio (MLR) and overall survival of patients with Gallbladder carcinoma (GBC).

\begin{tabular}{|c|c|c|}
\hline Study & & $\mathrm{HR}(95 \% \mathrm{CI})$ \\
\hline Zhang 2015 & 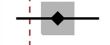 & $1.74(1.18,2.56)$ \\
\hline Cui 2018 & & $1.24(0.87,1.75)$ \\
\hline Tao 2018 & 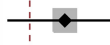 & $1.86(1.09,3.18)$ \\
\hline Du 2018 & & $0.75(0.35,1.06)$ \\
\hline Choi 2019 & $\stackrel{\leftrightarrow}{\longrightarrow}$ & $1.70(1.09,2.63)$ \\
\hline Deng 2019 & & $1.22(0.79,1.90)$ \\
\hline Navarro 2019 & & $0.53(0.11,2.55)$ \\
\hline You 2019 & 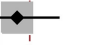 & $1.19(0.79,1.77)$ \\
\hline Overall $(\mathrm{I}-$ squared $=33.4 \%, \mathrm{p}=0.162)$ & \langle & $1.34(1.14,1.57)$ \\
\hline Test of ES $=1: z=3.55 p=0.000$ & & \\
\hline
\end{tabular}

FIGURE 4 | Forest plot for the association between platelet-to-lymphocyte ratio (PLR) and overall survival of patients with Gallbladder carcinoma (GBC).

preoperative applicability etc. $(7,8)$. Although there were growing evidence of using these markers to predict survival in various cancers, no worldwide consensus has been achieved and concerns rise about the efficiency and accuracy of these makers, and thus their clinical utilities are still suspended and limited.

In the present review, we focused on the prognostic value of one series of the most easily accessible and investigated markers that is WBCC-derived indexes including NLR, LMR/MLR and PLR in GBC patients. Our meta-analysis showed that all these indexes could be used as prognostic factors for GBC patients, which was in compliance with results in other cancer types (31). And we also pooled available data together and revealed that elevated NLR was significantly correlated with TNM stage, tumor differentiation and CA19-9, which could explain in part the mechanisms of these indexes being used as prognostic markers for GBC patients.

Although WBCC-derived indexes are certified to be useful parameters for predicting prognosis in various cancer types, the underlying mechanisms largely remains to be elucidated. First of all, the theoretical foundation of their usage as prognostic biomarker lies in that different types of peripheral blood cells could be considered to reflect host immune and inflammation status, which play important role in systemic inflammatory response (SIR), carcinogenesis, tumor microenvironment 
A

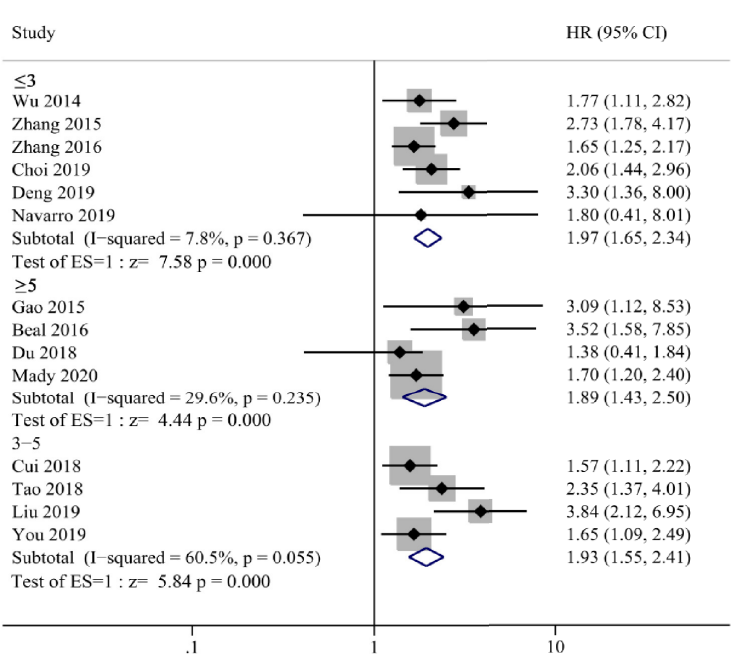

B

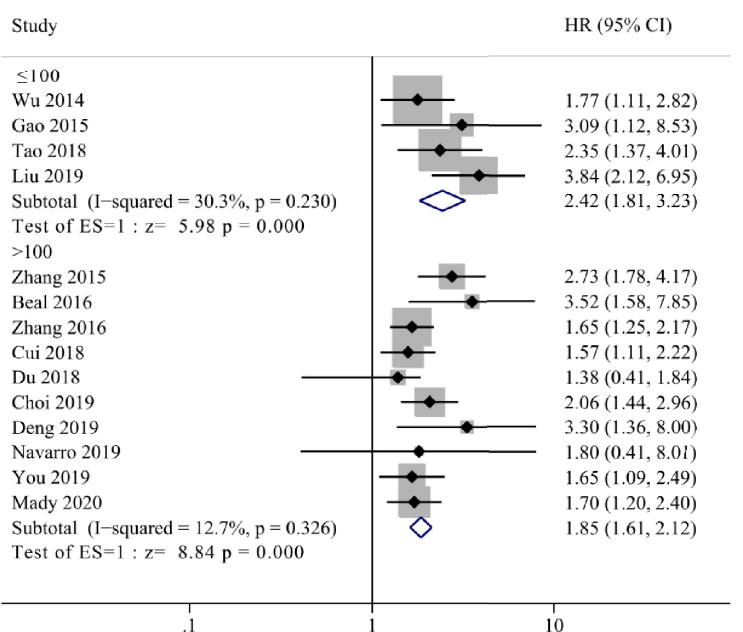

C

Study $\quad$ HR $(95 \% \mathrm{Cl})$

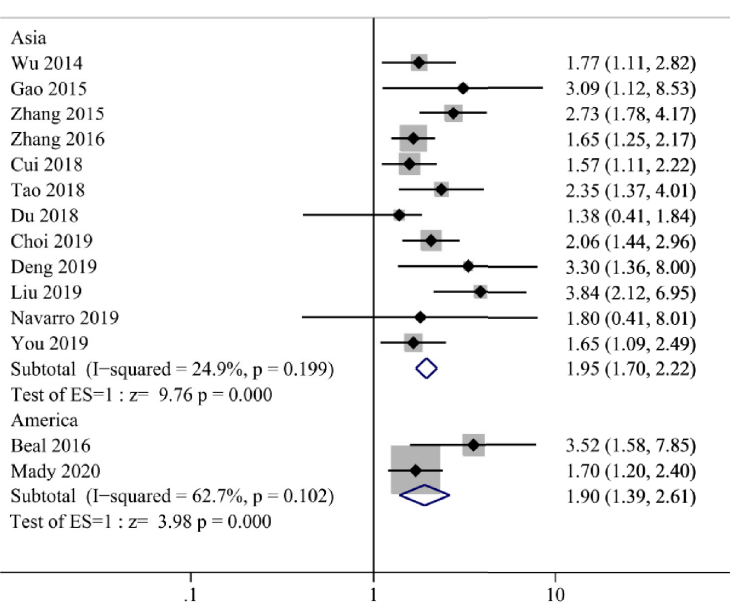

FIGURE 5 | Forest plot and subgroup analysis for (A) cut-off value, (B) sample size and (C) geographic area of the correlation between neutrophil-lymphocyte ratio (NLR) and overall survival of patients with Gallbladder carcinoma (GBC).

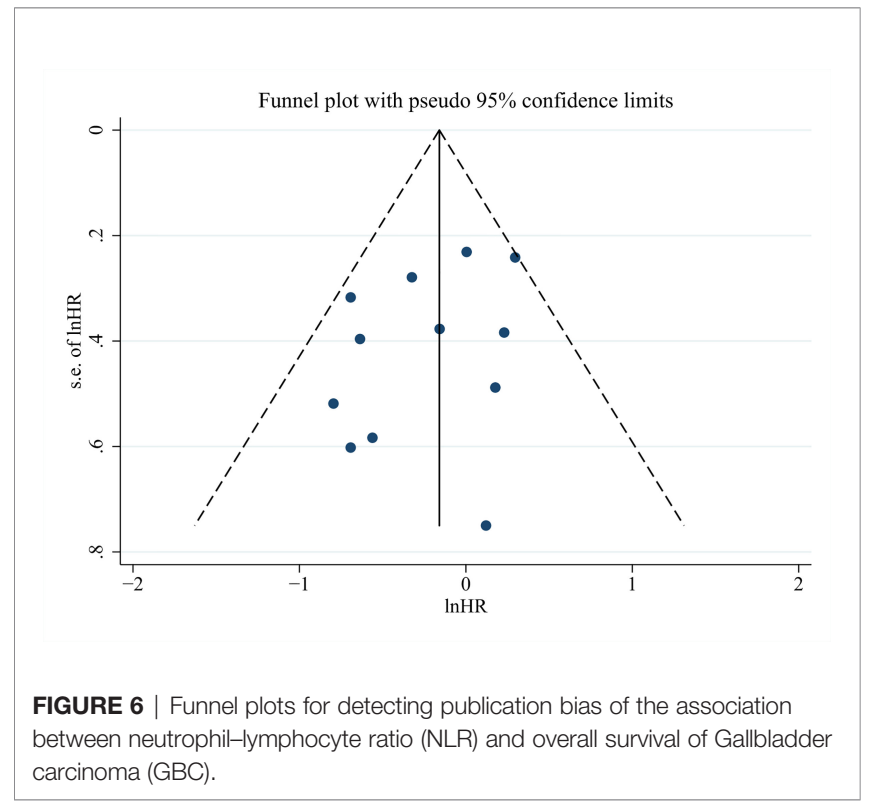

modulation and progression (40-42). Indeed, inflammatory microenvironment has been proposed as one hallmark of cancer (43), infiltrating immune and inflammatory cells are increasingly accepted to be generic constituents of tumors, and they exert conflicting ways for tumor development: tumorantagonizing effect as for lymphocytes while tumor-promoting effect as for neutrophils and monocytes $(37,39)$. More specifically, infiltrating lymphocytes are major antitumor components that may induce cancer cell apoptosis via interaction of $\mathrm{CD}^{+}$and $\mathrm{CD}^{+} \mathrm{T}$ cells $(44,45)$. In contrast, low lymphocytes within or around tumor area may be responsible for an insufficient immune surveillance that leads to tumor progression and inferior survival of patient (44). On the other hand, neutrophils and monocytes play a tumor-promoting role in malignancies. In short, neutrophils, as another key component of SIR, are recruited to tumor area, produce various kinds of cytokines and chemokines that are implicated in promoting tumor progression via all kinds of pathophysiological process, such as matrix degradation, immunosuppression, angiogenesis etc. $(43,46,47)$. Besides, peripheral monocytes are known for their association with the level of tumor-associated macrophages (TAMs) which could also produce cytokines and enzymes with protumoral functions, including tumor cell migration, invasion, metastasis as well as immunosuppression $(48,49)$. On the other hand, Deng and collogues revealed in their study that SIR biomarkers were significantly correlated with tumor differentiation, TNM stage or anemia, this could partially explain the positive correlation between SIR biomarkers and prognosis (17).

As we summarized above, most studies concluded with a favorable and positive results on prognosis predictive value of these WBCC-related biomarkers, but there are some limitations in these studies. Firstly, the cut-off value was discrepant through studies and different methods were employed to get it defined (Table 1). The receiver operating characteristic (ROC) curve 
analysis was the mostly adopted method and the accuracy of it was determined by sample size and subjective populations (as parameters vary among different tumors, stage, treatment etc.) (50). So future large volume investigations among different populations should be considered to identify the optimal cutoff values for each index. Secondly, the WBCC parameters are continuous variables which may present quite different values, especially before and after treatment. For example, the neutrophils are more susceptible to antibiotics usage, and may differ greatly from before and after surgery. So dynamic observations at different time point or analysis on trend should be more significant and encouraged for future investigations. Thirdly and finally, it is impossible to have one index fit to all situations, and the prognostic value of each index varies between different tumors, and even between different stages and treatment strategies in same tumor. So, the accuracy and reliability of single index may be challenged and an optimized mathematical model should provide some benefits to solve this issue. For example, Deng and collogues proposed a predictive nomogram using all the significant independent predicators to predict the patient survival (17). Each variable could be assigned a weighted number of points in the model, and the sum of points for each patient could be used to predict prognosis.

There were some limitations in this meta-analysis. Firstly, all studies were retrospective with low quality of evidence, further high quality RCT studies should be designed for future investigations. Secondly, the cut-off value was different through studies. Although we performed sub-group analyses, a single defined cut-off value would provide better comparison between studies. Thirdly, although there was no significant heterogeneity among most included studies, the patient inclusion criteria varied through studies, such as operation method, tumor staging, chemotherapy strategy etc. Due to the limited sample size, subgroup analysis was not practicable. Finally, all the included 14 studies investigated NLR as prognosis marker for GBC, while only few studies investigated MLR, PLR, so the statistics for these latter meta-analyses are under-powered, and

\section{REFERENCES}

1. Kakaei F, Beheshtirouy S, Nejatollahi SM, Zarrintan S, Mafi MR. Surgical Treatment of Gallbladder Carcinoma: A Critical Review. Updates Surg (2015) 67(4):339-51. doi: 10.1007/s13304-015-0328-x

2. WC L, Yun Z, S W, L J, WL F, XP G, et al. Analysis of the Incidence and Epidemic Trend of Gallbladder Cancer in China. Chin J Digestive Surg (2019) 18(2):186-9. doi: 10.3760/cma.j.issn.1673-9752.2019.02.015

3. Aloia TA, Jarufe N, Javle M, Maithel SK, Roa JC, Adsay V, et al. Gallbladder Cancer: Expert Consensus Statement. HPB (Oxford) (2015) 17(8):681-90. doi: 10.1111/hpb.12444

4. Jang JY, Heo JS, Han Y, Chang J, Kim JR, Kim H, et al. Impact of Type of Surgery on Survival Outcome in Patients With Early Gallbladder Cancer in the Era of Minimally Invasive Surgery: Oncologic Safety of Laparoscopic Surgery. Med (Baltimore) (2016) 95(22):e3675. doi: 10.1097/MD.0000000000003675

5. Hundal R, Shaffer EA. Gallbladder Cancer: Epidemiology and Outcome. Clin Epidemiol (2014) 6:99-109. doi: 10.2147/CLEP.S37357

6. Brierley J, Gospodarowicz MK, Wittekind C. TNM Classification of Malignant Tumours. Eighth edition. Chichester, West Sussex, UK; Hoboken, NJ: John Wiley \& Sons, Inc (2017).

7. Dolan RD, Laird BJA, Horgan PG, McMillan DC. The Prognostic Value of the Systemic Inflammatory Response in Randomised Clinical Trials in Cancer: A future studies on these markers should be expected and included for further meta-analysis.

In conclusion, our meta-analysis validated that WBCCderived indexes including NLR, MLR/LMR and PLR could be used as prognostic parameters for predicting survival outcomes in GBC patients. These series of indexes, especially NLR, could improve risk stratification and facilitate better patient selection for surgical resection or aggressive chemotherapy in the management of GBC.

\section{DATA AVAILABILITY STATEMENT}

The original contributions presented in the study are included in the article/supplementary material. Further inquiries can be directed to the corresponding authors.

\section{AUTHOR CONTRIBUTIONS}

Study concepts: TL, ZC and BX. Study design: TL, ZC and BX. Data acquisition: BX, JC, ZC and WZ. Quality control of data and algorithms: BX, JC, ZD and XZ. Data analysis and interpretation: BX, ZC and JC. Statistical analysis: ZC, BX, JZ, JC and WZ. Manuscript preparation: BX, ZC, JZ and JC. Manuscript editing: ZC, BX and JZ. Manuscript review: TL, $\mathrm{ZC}$ and BX. All authors contributed to the article and approved the submitted version.

\section{FUNDING}

This study was supported by the Taishan Scholars Program for Young Experts of Shandong Province (tsqn20161064), the National Natural Science Foundation of China (81874178 \& 82073200) for TL.

Systematic Review. Crit Rev Oncol Hematol (2018) 132:130-7. doi: 10.1016/ j.critrevonc.2018.09.016

8. Roxburgh CS, McMillan DC. Role of Systemic Inflammatory Response in Predicting Survival in Patients With Primary Operable Cancer. Future Oncol (2010) 6(1):149-63. doi: 10.2217/fon.09.136

9. Wu XS, Shi LB, Li ML, Ding Q, Weng H, Wu WG, et al. Evaluation of Two Inflammation-Based Prognostic Scores in Patients With Resectable Gallbladder Carcinoma. Ann Surg Oncol (2014) 21(2):449-57. doi: 10.1245/s10434-013-3292-Z

10. Gao H, Zhang H, Xu X, Zou W, Lin K, Deng Y, et al. Neutrophil-Lymphocyte Ratio as a Prognostic Factor for Gallbladder Carcinoma After Radical Resection. World Chin J Digestol (2015) 23(35):5705. doi: 10.11569/wcjd.v23.i35.5705

11. Zhang Y, Jiang C, Li J, Sun J, Qu X. Prognostic Significance of Preoperative Neutrophil/Lymphocyte Ratio and Platelet/Lymphocyte Ratio in Patients With Gallbladder Carcinoma. Clin Transl Oncol (2015) 17(10):810-8. doi: 10.1007/s12094-015-1310-2

12. Beal EW, Wei L, Ethun CG, Black SM, Dillhoff M, Salem A, et al. Elevated NLR in Gallbladder Cancer and Cholangiocarcinoma - Making Bad Cancers Even Worse: Results From the US Extrahepatic Biliary Malignancy Consortium. HPB (Oxford) (2016) 18(11):950-7. doi: 10.1016/j.hpb.2016.08.006

13. Zhang L, Wang R, Chen W, Xu X, Dong S, Fan H, et al. Prognostic Significance of Neutrophil to Lymphocyte Ratio in Patients With Gallbladder Carcinoma. HPB (Oxford) (2016) 18(7):600-7. doi: 10.1016/j.hpb.2016.03.608 
14. Cui X, Zhu S, Tao Z, Deng X, Wang Y, Gao Y, et al. Long-Term Outcomes and Prognostic Markers in Gallbladder Cancer. Med (Baltimore) (2018) 97(28): e11396. doi: 10.1097/MD.0000000000011396

15. Tao Z, Li SX, Cui X, Huang Y, Zhu S, Wang Y, et al. The Prognostic Value of Preoperative Inflammatory Indexes in Gallbladder Carcinoma With Hepatic Involvement. Cancer Biomark (2018) 22(3):551-7. doi: 10.3233/CBM-181230

16. Choi YH, Lee JW, Lee SH, Choi JH, Kang J, Lee BS, et al. A High Monocyte-toLymphocyte Ratio Predicts Poor Prognosis in Patients With Advanced Gallbladder Cancer Receiving Chemotherapy. Cancer Epidemiol Biomarkers Prev (2019) 28(6):1045-51. doi: 10.1158/1055-9965.EPI-18-1066

17. Deng Y, Zhang F, Yu X, Huo CL, Sun ZG, Wang S, et al. Prognostic Value of Preoperative Systemic Inflammatory Biomarkers In Patients With Gallbladder Cancer and The Establishment of A Nomogram. Cancer Manag Res (2019) 11:9025-35. doi: 10.2147/CMAR.S218119

18. Du JH, Lu J. Circulating CEA-dNLR Score Predicts Clinical Outcome of Metastatic Gallbladder Cancer Patient. J Clin Lab Anal (2019) 33(2):e22684. doi: $10.1002 /$ jcla.22684

19. Liu F, Hu HJ, Ma WJ, Yang Q, Wang JK, Li FY, et al. Prognostic Significance of Neutrophil-Lymphocyte Ratio and Carbohydrate Antigen 19-9 in Patients With Gallbladder Carcinoma. Med (Baltimore) (2019) 98(8):e14550. doi: 10.1097/MD.0000000000014550

20. You MS, Ryu JK, Choi YH, Choi JH, Huh G, Paik WH, et al. Therapeutic Outcomes and Prognostic Factors in Unresectable Gallbladder Cancer Treated With Gemcitabine Plus Cisplatin. BMC Cancer (2019) 19(1):10. doi: 10.1186/s12885-018-5211-y

21. Navarro J, Kang I, Hwang HK, Yoon DS, Lee WJ, Kang CM. Glucose to Lymphocyte Ratio as a Prognostic Marker in Patients With Resected Pt2 Gallbladder Cancer. J Surg Res (2019) 240:17-29. doi: 10.1016/j.jss.2019.02.043

22. Mady M, Prasai K, Tella SH, Yadav S, Hallemeier CL, Rakshit S, et al. Neutrophil to Lymphocyte Ratio as a Prognostic Marker in Metastatic Gallbladder Cancer. HPB (2020) 22(10):1490-5. doi: 10.1016/j.hpb.2020.02.002

23. Shea BJ, Reeves BC, Wells G, Thuku M, Hamel C, Moran J, et al. AMSTAR 2: A Critical Appraisal Tool for Systematic Reviews That Include Randomised or non-Randomised Studies of Healthcare Interventions, or Both. BMJ (2017) 358:j4008. doi: 10.1136/bmj.j4008

24. Moher D, Liberati A, Tetzlaff J, Altman DG, Group P. Preferred Reporting Items for Systematic Reviews and Meta-Analyses: The PRISMA Statement. BMJ (2009) 339:b2535. doi: 10.1136/bmj.b2535

25. Michiels S, Piedbois P, Burdett S, Syz N, Stewart L, Pignon JP. Meta-Analysis When Only the Median Survival Times Are Known: A Comparison With Individual Patient Data Results. Int J Technol Assess Health Care (2005) 21 (1):119-25. doi: 10.1017/S0266462305050154

26. Jeng KS, Ohta I, Yang FS. Reappraisal of the Systematic Management of Complicated Hepatolithiasis With Bilateral Intrahepatic Biliary Strictures. Arch Surg (1996) 131(2):141-7. doi: 10.1001/archsurg.1996.01430140031008

27. Wan X, Wang W, Liu J, Tong T. Estimating the Sample Mean and Standard Deviation From the Sample Size, Median, Range and/or Interquartile Range. BMC Med Res Methodol (2014) 14:135. doi: 10.1186/1471-2288-14-135

28. Luo D, Wan X, Liu J, Tong T. Optimally Estimating the Sample Mean From the Sample Size, Median, Mid-Range, and/or Mid-Quartile Range. Stat Methods Med Res (2018) 27(6):1785-805. doi: 10.1177/0962280216669183

29. Bray F, Ferlay J, Soerjomataram I, Siegel RL, Torre LA, Jemal A. Global Cancer Statistics 2018: GLOBOCAN Estimates of Incidence and Mortality Worldwide for 36 Cancers in 185 Countries. CA Cancer J Clin (2018) 68(6):394-424. doi: $10.3322 /$ caac. 21492

30. Sharma A, Sharma KL, Gupta A, Yadav A, Kumar A. Gallbladder Cancer Epidemiology, Pathogenesis and Molecular Genetics: Recent Update. World J Gastroenterol (2017) 23(22):3978-98. doi: 10.3748/wjg.v23.i22.3978

31. Paramanathan A, Saxena A, Morris DL. A Systematic Review and MetaAnalysis on the Impact of Pre-Operative Neutrophil Lymphocyte Ratio on Long Term Outcomes After Curative Intent Resection of Solid Tumours. Surg Oncol (2014) 23(1):31-9. doi: 10.1016/j.suronc.2013.12.001

32. Chua W, Charles KA, Baracos VE, Clarke SJ. Neutrophil/Lymphocyte Ratio Predicts Chemotherapy Outcomes in Patients With Advanced Colorectal Cancer. Br J Cancer (2011) 104(8):1288-95. doi: 10.1038/bjc.2011.100

33. Shimose S, Kawaguchi T, Iwamoto H, Tanaka M, Miyazaki K, Ono M, et al. Controlling Nutritional Status (CONUT) Score Is Associated With Overall
Survival in Patients With Unresectable Hepatocellular Carcinoma Treated With Lenvatinib: A Multicenter Cohort Study. Nutrients (2020) 12(4):1076. doi: 10.3390/nu12041076

34. Liang RF, Li JH, Li M, Yang Y, Liu YH. The Prognostic Role of Controlling Nutritional Status Scores in Patients With Solid Tumors. Clin Chim Acta (2017) 474:155-8. doi: 10.1016/j.cca.2017.09.021

35. Lee JY, Kim HI, Kim YN, Hong JH, Alshomimi S, An JY, et al. Clinical Significance of the Prognostic Nutritional Index for Predicting Short- and Long-Term Surgical Outcomes After Gastrectomy: A Retrospective Analysis of 7781 Gastric Cancer Patients. Med (Baltimore) (2016) 95(18):e3539. doi: 10.1097/MD.0000000000003539

36. Li SQ, Jiang YH, Lin J, Zhang J, Sun F, Gao QF, et al. Albumin-to-Fibrinogen Ratio as a Promising Biomarker to Predict Clinical Outcome of Non-Small Cell Lung Cancer Individuals. Cancer Med (2018) 7(4):1221-31. doi: 10.1002/cam4.1428

37. Saqib R, Pathak S, Smart N, Nunes Q, Rees J, Finch Jones M, et al. Prognostic Significance of Pre-Operative Inflammatory Markers in Resected Gallbladder Cancer: A Systematic Review. ANZ J Surg (2018) 88(6):554-9. doi: 10.1111/ ans. 14300

38. McMillan DC. The Systemic Inflammation-Based Glasgow Prognostic Score: A Decade of Experience in Patients With Cancer. Cancer Treat Rev (2013) 39 (5):534-40. doi: 10.1016/j.ctrv.2012.08.003

39. Yang R, Chang Q, Meng X, Gao N, Wang W. Prognostic Value of Systemic Immune-Inflammation Index in Cancer: A Meta-Analysis. J Cancer (2018) 9 (18):3295-302. doi: 10.7150/jca.25691

40. Grivennikov SI, Greten FR, Karin M. Immunity, Inflammation, and Cancer. Cell (2010) 140(6):883-99. doi: 10.1016/j.cell.2010.01.025

41. Coussens LM, Werb Z. Inflammation and Cancer. Nature (2002) 420 (6917):860-7. doi: 10.1038/nature01322

42. Diakos CI, Charles KA, McMillan DS, Clarke SJ. Cancer-Related Inflammation and Treatment Effectiveness. Lancet Oncol (2014) 15(11): e493-503. doi: 10.1016/S1470-2045(14)70263-3

43. Hanahan D, Weinberg RA. Hallmarks of Cancer: The Next Generation. Cell (2011) 144(5):646-74. doi: 10.1016/j.cell.2011.02.013

44. Pages F, Galon J, Dieu-Nosjean MC, Tartour E, Sautes-Fridman C, Fridman WH. Immune Infiltration in Human Tumors: A Prognostic Factor That Should Not be Ignored. Oncogene (2010) 29(8):1093-102. doi: 10.1038/onc.2009.416

45. Dunn GP, Old LJ, Schreiber RD. The Immunobiology of Cancer Immunosurveillance and Immunoediting. Immunity (2004) 21(2):137-48. doi: 10.1016/j.immuni.2004.07.017

46. Gregory AD, Houghton AM. Tumor-Associated Neutrophils: New Targets for Cancer Therapy. Cancer Res (2011) 71(7):2411-6. doi: 10.1158/00085472.CAN-10-2583

47. Imai Y, Kubota Y, Yamamoto S, Tsuji K, Shimatani M, Shibatani N, et al. Neutrophils Enhance Invasion Activity of Human Cholangiocellular Carcinoma and Hepatocellular Carcinoma Cells: An In Vitro Study. J Gastroenterol Hepatol (2005) 20(2):287-93. doi: 10.1111/j.1440-1746.2004.03575.x

48. Franklin RA, Liao W, Sarkar A, Kim MV, Bivona MR, Liu K, et al. The Cellular and Molecular Origin of Tumor-Associated Macrophages. Science (2014) 344(6186):921-5. doi: 10.1126/science.1252510

49. Condeelis J, Pollard JW. Macrophages: Obligate Partners for Tumor Cell Migration, Invasion, and Metastasis. Cell (2006) 124(2):263-6. doi: 10.1016/ j.cell.2006.01.007

50. Walker SP. The ROC Curve Redefined - Optimizing Sensitivity (and Specificity) to the Lived Reality of Cancer. N Engl J Med (2019) 380(17):1594-5. doi: 10.1056/NEJMp1814951

Conflict of Interest: The authors declare that the research was conducted in the absence of any commercial or financial relationships that could be construed as a potential conflict of interest.

Copyright $\odot 2021 \mathrm{Xu}$, Chen, Zhang, Chang, Zhao, Dong, Zhi and Li. This is an openaccess article distributed under the terms of the Creative Commons Attribution License (CC BY). The use, distribution or reproduction in other forums is permitted, provided the original author(s) and the copyright owner(s) are credited and that the original publication in this journal is cited, in accordance with accepted academic practice. No use, distribution or reproduction is permitted which does not comply with these terms. 